ULB-PMIF-93/11

\title{
Renormalization of gauge invariant operators and anomalies in Yang-Mills theory
}

\author{
Glenn Barnich ${ }^{\dagger}$ and Marc Henneaux* \\ Faculté des Sciences, Université Libre de Bruxelles, \\ Campus Plaine C.P. 231, B-1050 Bruxelles, Belgium
}

\begin{abstract}
A long-standing conjecture on the structure of renormalized, gauge invariant, integrated operators of arbitrary dimension in Yang-Mills theory is established. The general solution of the consistency condition for anomalies with sources included is also derived. This is achieved by computing explicitely the cohomology of the full unrestricted BRST operator in the space of local polynomial functionals with ghost number equal to zero or one. The argument does not use power counting and is purely cohomological. It relies crucially on standard properties of the antifield formalism.
\end{abstract}

$\left.{ }^{\dagger}\right)$ Aspirant au Fonds National de la Recherche Scientifique (Belgium)

$\left.{ }^{*}\right)$ Also at Centro de Estudios Científicos de Santiago, Chile. 
The antifield formalism [1] is well known to be extremely useful in the study of theories with a complicated off-shell gauge structure like supergravity or string field theory. Much less appreciated is the fact that the ideas underlying the antifield construction -in particular the central feature that the antifields implement the equations of motion in cohomology [2]- are actually quite helpful already in the analysis of the Yang-Mills field. An illustration of this property has been given in [3], where the demonstration of an old theorem by Joglekar and Lee [4] on the renormalization of local gauge invariant operators was streamlined using concepts from the antifield formalism. In this letter, we extend the analysis of [3] by proving a long-standing conjecture originally due to Kluberg-Stern and Zuber [5, 6, 7] concerning the structure of renormalized, integrated, gauge invariant operators (like $\operatorname{tr} F_{\mu \nu}^{2}$ at zero momentum, i.e., $\left.\int \operatorname{tr} F_{\mu \nu}^{2} d^{4} x\right)$. We also provide the general solution of the anomaly equation with sources included. Our approach does not use power counting and is purely cohomological. Hence, it is valid for operators of arbitrarily high physical dimension, for which our results control both the possible counterterms and the anomalies.

Thanks to the effort of various people over many years, it has been shown that most questions about the quantum properties of Yang-Mills models can be reformulated as algebraic questions involving graded differential algebras. It would be out of place to review here the huge body of work that has gone into that problem. We shall rather refer to the recent historical survey given in reference [8].

As recalled there, the analysis of renormalized, integrated, gauge invariant operators and of anomalies ultimately boils down to the calculation of the cohomology of the BRST differential $s$ in the space of integrated polynomials in the Yang-Mills potential $A_{\mu}^{a}$, the ghosts $C^{a}$, the matter fields $y^{i}$ (belonging to some representation of the gauge group), the antifields $A_{a}^{* \mu}, C_{a}^{*}$, and $y_{i}^{*}$ (also called "sources for the BRST variations"), as well as their spacetime derivatives. More precisely, the question is to find the general solution of the equation

$$
s A=0,
$$

where $s$ is the BRST differential and where $A$ is the integral of a polynomial in $A_{\mu}^{a}, C^{a}, y^{i}, A_{a}^{* \mu}, C_{a}^{*}, y_{i}^{*}$ and their derivatives of ghost number zero or one,

$$
A=\int a d^{4} x, \quad g h A=0 \text { or } 1 .
$$


We shall allow for the presence of abelian factors in the gauge group but we shall assume, however, that each abelian gauge field is coupled to at least one charged matter field (no free abelian gauge field). The following theorems are the central results reported in this letter.

Theorem 1 : the general solution of (1) with ghost number zero is given by

$$
A=\int \bar{a} d^{4} x+s \int b d^{4} x
$$

where $\bar{a}$ is an invariant polynomial in the field strengths, the matter fields, and their covariant derivatives. If the gauge group has abelian factors, there are in addition extra solutions given by

$$
A=k_{A}^{\Delta} \int\left(j_{\Delta}^{\mu} A_{\mu}^{A}+t_{\Delta \mu}^{a} A_{a}^{* \mu} C^{A}+t_{\Delta}^{i} y_{i}^{*} C^{A}\right) d^{4} x
$$

where: (i) $k_{A}^{\Delta}$ are arbitrary constants ; (ii) $A_{\mu}^{A}$ are the abelian gauge fields and $C^{A}$ the abelian ghosts; and (iii) $j_{\Delta}^{\mu}$ are gauge invariant conserved currents (for each $\Delta$ ),

$$
\partial_{\mu} j_{\Delta}^{\mu}=t_{\Delta \mu}^{a} \frac{\delta \mathcal{L}}{\delta A_{\mu}^{a}}+t_{\Delta}^{i} \frac{\delta \mathcal{L}}{\delta y^{i}}
$$

Theorem 2 : the general solution of (1) with ghost number one is given by

$$
A=\int c_{1} d^{4} x+s \int b d^{4} x
$$

with

$$
c_{1}=\operatorname{tr}\left(C \partial_{\mu} A_{\nu} \partial_{\lambda} A_{\rho}+\frac{3}{2} C \partial_{\mu} A_{\nu} A_{\lambda} A_{\rho}\right) \varepsilon^{\mu \nu \lambda \rho},
$$

(Adler-Bardeen-Bell-Jackiw anomaly). Again, if there are abelian factors, there are further solutions and one must replace (6) by

$$
\begin{array}{r}
A=\int c_{1} d^{4} x+\int \mu_{A} C^{A} d^{4} x+\int c_{2} d^{4} x+ \\
\int c_{3} d^{4} x+s \int b d^{4} x
\end{array}
$$


where (i) $\mu_{A}$ are invariant polynomials in the field strengths, the matter fields and their covariant derivatives ; (ii) $c_{2}$ does not depend on the antifields

$$
c_{2}=k_{A} F_{\mu \nu}^{A} \operatorname{tr}\left(C \partial_{\lambda} A_{\rho}\right) \varepsilon^{\mu \nu \lambda \rho}
$$

and (iii) $c_{3}$ is given by

$$
\begin{aligned}
c_{3}= & k_{A B}^{\Delta}\left(j_{\Delta}^{\mu} A_{\mu}^{A} C^{B}+\frac{1}{2} t_{\Delta \mu}^{a} A_{a}^{* \mu} C^{A} C^{B}\right. \\
& \left.+\frac{1}{2} t_{\Delta}^{i} y_{i}^{*} C^{A} C^{B}\right), \quad k_{A B}^{\Delta}=-k_{B A}^{\Delta} .
\end{aligned}
$$

As in (4), the $j_{\Delta}^{\mu}$ are conserved currents.

The solution (3) is BRST-trivial if and only if $\bar{a}$ vanishes on-shell (the antifields enable one to rewrite a polynomial that vanishes on-shell as a $s$ variation). The solutions (4) and (10) are trivial if and only if the conserved current $j_{\Delta}^{\mu}$ is trivial, i.e., equal on-shell to an identically conserved total divergence. The conjecture of Kluberg-Stern and Zuber mentioned above follows from a direct inspection of the solutions given in theorems 1 and 2 :

Corollary 1 : for a semi-simple gauge group, the general solution of $s \int a d^{4} x=0$ with ghost number zero is, up to s-boundaries (i.e., s-exact terms), equal to the integral of a gauge invariant polynomial in the field strengths, the matter fields and their covariant derivatives.

Corollary 2 : similarily, the only possible anomaly for a semi-simple gauge group is of the Adler-Bardeen-Bell-Jackiw type.

Thus, the conjecture holds for QCD or grand unified models. However, if the gauge group has abelian factors, like in the standard model with $U(1) \times$ $S U(2) \times S U(3)$, then there are further non-trivial solutions to $s \int a d^{4} x=0$ besides (3) and (6), (7). The anomaly solution (9), which does not depend on the antifields, has been known for some time. The other extra solutions depend explicitly on the antifields and deserve some comments.

(i) All the antifield dependent solutions are completely determined (in cohomology) by their antifield independent component $A_{0}$, which is a solution of $s A_{0} \approx 0$ where $\approx$ means equal modulo the equations of motion). 
This is a standard result of homological perturbation theory, which indicates how the antifield dependent components follow recursively from the antifield independent ones (see e.g. [9], chapter 8). Thus, all the information about (4) or (10) is respectively contained in $k_{A}^{\Delta} j_{\Delta}^{\mu} A_{\mu}^{A}$ or $k_{A B}^{\Delta} j_{\Delta}^{\mu} A_{\mu}^{A} C^{B}$.

(ii) The solutions (4) and (10) involve explicitly conserved currents. An example of (10) has been given in 10. While (10) needs at least two abelian factors, the solution () exists already with a single $U(1)$ provided there are non trivial conserved currents. By the Noether theorem, a non trivial conserved current exists for each non trivial rigid symmetry (e.g., rigid $S U(N)$ flavor symmetry). If all exact symmetries are gauge symmetries, however, all the conserved currents are trivial and the solutions (4) and (10) are also trivial. If the spacetime symmetries are not gauged, one may take for $j_{\Delta}^{\mu}$ the energy momentum tensor $T_{\nu}^{\mu}$ (but the solution is then non covariant). It is not surprising that the solutions of $s A=0$ contain information about the dynamics through the appearance of conserved currents, since the role of the antifields is just to implement the equations of motion in cohomology ([2, 9]).

(iii) Antifield dependent solutions of $s A=0$ exist for simple gauge groups but only in ghost degree $\geq 2$. For instance, $\int j^{\mu} \operatorname{tr}\left(A_{\mu} C^{2}\right) d^{4} x+$ antifield dependent terms is such a solution -which involves again a conserved current.

(iv) Although of mathematical interest, it is not known whether the solutions (4) and (10) do arise in practice in the renormalization of integrated, gauge invariant operators. An example where an antifield-independent solution of the type $\mu_{A} C^{A}$ in (8) occurs is given in [11.

(v) If there are free abelian gauge fields, the equation (1) admits further solutions. This case is rather academical in the present context but is of interest when one considers the problem of introducing consistent couplings among free, spin 1 gauge fields [12]. The extra solutions correspond to nonabelian deformations of the abelian theory. They are not written here for the sake of brievity but will be discussed elsewhere [13].

We now pass to the demonstration of the theorems. The BRST differential in Yang-Mills theory is a sum of two differentials,

$$
s=\delta+\gamma
$$

Since both $\delta$ and $\gamma$ are derivations and commute with $\partial_{\mu}$, it is enough to 
define $\delta$ and $\gamma$ on the generators $A_{\mu}^{a}, C^{a}, y^{i}, A_{a}^{* \mu}, C_{a}^{*}$ and $y_{i}^{*}$ 14. One has

$$
\begin{array}{r}
\delta A_{\mu}^{a}=0, \delta C^{a}=0, \delta y^{i}=0 \\
\delta A_{a}^{* \mu}=\frac{\delta \mathcal{L}}{\delta A_{\mu}^{a}}, \delta C_{a}^{*}=D_{\mu} A_{a}^{* \mu}-T_{a i}^{j} y_{j}^{*} y^{i}, \delta y_{i}^{*}=\frac{\delta \mathcal{L}}{\delta y^{i}}
\end{array}
$$

and

$$
\begin{array}{r}
\gamma A_{\mu}^{a}=D_{\mu} C^{a}, \gamma C^{a}=\frac{1}{2} C_{b c}^{a} C^{b} C^{c}, \gamma y^{i}=T_{a j}^{i} y^{j} C^{a} \\
\gamma A_{a}^{* \mu}=-A_{c}^{* \mu} C_{a b}^{c} C^{b}, \gamma C_{a}^{*}=-C_{c}^{*} C_{a b}^{c} C^{b} \\
\gamma y_{i}^{*}=-T_{a i}^{j} y_{j}^{*} C^{a}
\end{array}
$$

where the $T_{a}$ 's are the generators of the representation to which the $y^{i}$ belong. This implies

$$
\delta^{2}=0, \gamma^{2}=0, \gamma \delta+\delta \gamma=0 .
$$

The decomposition (11) of $s$ is quite standard from the point of view of the antifield formalism [2, 9]. It corresponds to the introduction of a further grading besides the ghost number,which is called the "antighost" (or "antifield") number and is denoted by antigh,

$$
\begin{array}{r}
\text { antigh } A_{\mu}^{a}=0, \text { antigh } C^{a}=0, \text { antigh } y^{i}=0 \\
\text { antigh } A_{a}^{* \mu}=1, \text { antigh } C_{a}^{*}=2, \text { antigh } y_{i}^{*}=1 \\
\text { antigh } \delta=-1, \text { antigh } \gamma=0
\end{array}
$$

(One has also, of course, $g h A_{\mu}^{a}=0, g h C^{a}=1, g h y^{i}=0, g h A_{a}^{* \mu}=-1$, $\left.g h C_{a}^{*}=-2, g h y_{i}^{*}=-1, g h \delta=g h \gamma=g h s=1\right)$. The differential $s$ is sometimes called the full, unrestricted BRST differential, while the differential $\gamma$ acting on the polynomials in $A_{\mu}^{a}, C^{a}$ and their derivatives (but no antifields), is called the restricted BRST differential [15]. The differential $\delta$ is the "Koszul-Tate differential" and provides a resolution of the algebra of functions on the stationary surface where the equations of motion hold [2, 9].

The first step in the proofs of theorems 1 and 2 amounts to rewriting the condition $s \int a d^{4} x=0$ in terms of the integrand $a$. To that end, it is convenient to adopt form notations and to replace the polynomial $a$ by the 4-form $a d x^{0} \wedge \ldots \wedge d^{3} x$, which we still denote by $a$. We shall call $\mathcal{B}$ the 
algebra of spacetime exterior forms with coefficients that are polynomials in $A_{\mu}^{a}, C^{a}, y^{i}, A_{a}^{* \mu}, C_{a}^{*}, y_{i}^{*}$ and their derivatives ; and we shall call $\mathcal{E}$ the algebra of spacetime exterior forms with coefficients that are polynomials in $A_{\mu}^{a}, C^{a}$, $y^{i}$ and their derivatives (no antifields).

Because of Stokes theorem ( $\int d b=0$-we assume as it is usual in this context, that all surface terms are zero), one can remove the integral sign at the price of allowing for the presence of a total exterior derivative. The condition $s \int a=0$ is equivalent to

$$
s a+d b=0 .
$$

And $\int a$ is exact if and only if

$$
a=s c+d e .
$$

Thus, the problem of computing the cohomology of $s$ in the space of integrated polynomials becomes that of computing the cohomology of $H^{*, 4}(s \mid d, \mathcal{B})$ of $s$ modulo $d$ in the algebra of polynomial forms of degree 4 . Note that the exterior derivative $d$ anticommutes with $\delta$ and $\gamma$ since $\left[\partial_{\mu}, \delta\right]=\left[\partial_{\mu}, \gamma\right]=0$.

A number of cohomologies related to $H^{*, 4}(s \mid d, \mathcal{B})$ have already been computed explicitely in the literature. These are $H^{*}(d, \mathcal{B}), H^{*}(\delta, \mathcal{B}), H^{*}(\gamma, \mathcal{E})$, $H^{*}(\gamma, \mathcal{B}), H^{*}(s, \mathcal{B}), H^{*}\left(d, H^{*}(\gamma, \mathcal{E})\right)$ and $H^{*}(\gamma \mid d, \mathcal{E})$. Of particular importance for the sequel is $H^{*}(\gamma \mid d, \mathcal{E})$ because any solution $a$ of $s a+d b=0$ which does not involve the antifields is automatically a solution of $\gamma a+d b=0$. And if it is trivial in $H^{*}(\gamma \mid d, \mathcal{E})$ (i.e., of the form $\gamma c+d e$ where $c$ and $e$ do not involve the antifields), then it is also trivial in $H^{*}(s \mid d, \mathcal{B})(\gamma c=s c) \llbracket 16 \rrbracket$. The strategy for computing $H^{*}(s \mid d, \mathcal{B})$ adopted here is to relate as much as possible elements of $H^{*}(s \mid d, \mathcal{B})$ to the known elements of $H^{*}(\gamma \mid d, \mathcal{E})$ 15, 17, 18 by eliminating the antifields. This is not always possible. But the equivalence classes of $H^{*}(s \mid d, \mathcal{B})$ with no representative in $\mathcal{E}$ are easily exhibited and correspond precisely to (4) and (10) above.

In order to control the antifield dependence of the solutions of (18), one needs two intermediate results.

Lemma 1 : let $b$ be a $\gamma$-closed form with degree $<4$ and antighost number $>0$. If $d b$ is $\gamma$-exact, then $b$ is trivial, i.e., $b=\gamma m+d n$ where $n$ is $\gamma$-closed. In other words,

$$
H_{k}^{l, j}\left(d, H^{*}(\gamma, \mathcal{B})\right)=0 \text { for } j<4 \text { and } k \geq 1 \text {. }
$$


Here and in the sequel, the first upper index $l$ is the ghost number, the second index $j$ is the form degree and the lower index $k$ is the antighost number. The lemma is proved exactly as the proposition on page 363 of [18].

The second intermediate result deals with $H_{k}^{l, j}(\delta \mid d)$. It is well known that $H_{k}^{l, j}(\delta)$ vanishes for $k \geq 1$ in $\mathcal{B}$ [19]. It turns out that the cohomological groups $H_{k}^{l, j}(\delta \mid d)$ with $k \geq 1$ are not zero, but only for $k=1$ and $j=4$. More precisely, one has

Lemma 2 : The group $H_{1}^{-1,4}(\delta \mid d)$ is isomorphic to the space of non trivial conserved currents. The other groups $H_{k}^{l, j}(\delta \mid d)$ with $k \geq 1$ all vanish.

This lemma will be proved in [13].

We can now demonstrate the theorems. Let $a$ be a 4-form solution of $s a+d b=0$ with ghost number equal to 0 or 1 . Expand $a$ and $b$ according to the antighost number,

$$
a=a_{0}+\ldots+a_{k}, \quad b=b_{0}+\ldots+b_{k} .
$$

Assume $k \geq 1$. The equation $s a+d b=0$ implies, at antighost number $k$, $\gamma a_{k}+d b_{k}=0$. By the standard descent argument, this equation yields the chain of equations $\gamma b_{k}+d b_{k}^{\prime}=0, \gamma b_{k}^{\prime}+d b_{k}^{\prime \prime}=0, \gamma b_{k}^{\prime \prime}+d b_{k}^{\prime \prime \prime}=0, \gamma b_{k}^{\prime \prime \prime}=0$ for some 2 -form $b_{k}^{\prime}$, 1-form $b_{k}^{\prime \prime}$ and 0 -form $b_{k}^{\prime \prime \prime}$. By lemma $1, b_{k}^{\prime \prime \prime}$ is trivial, $b_{k}^{\prime \prime \prime}=\gamma \rho_{k}$. But then one can make redefinitions so that it vanishes. Repeating the argument for $b_{k}^{\prime \prime}, b_{k}^{\prime}$ and $b_{k}$ shows that $b_{k}$ can be assumed to be equal to zero. Thus, $a_{k}$ is $\gamma$-closed. The general solution of $\gamma a_{k}=0$ is known [15, 17, 18, 20, 3] and reads, up to inessential $\gamma$-exact terms

$$
a_{k}=\Sigma a_{J} \omega^{J}(C)
$$

where $\omega^{J}(C)$ form a basis of the cohomology of the Lie algebra of the gauge group (invariant cocycles) and where $a_{J}$ are invariant polynomials in the field strengths, the matter fields, the antifields and their covariant derivatives. The last but one equation in $s a+d b=0$ reads $\gamma a_{k-1}+\delta a_{k}+d b_{k-1}=0$. Since $\gamma \delta a_{k}=-\delta \gamma a_{k}=0$, one gets again a descent for $b_{k-1}$, which reads $\gamma b_{k-1}+d b_{k-1}^{\prime}=0, \gamma b_{k-1}^{\prime}+d b_{k-1}^{\prime \prime}=0, \gamma b_{k-1}^{\prime \prime}+d b_{k-1}^{\prime \prime \prime}=0, \gamma b_{k-1}^{\prime \prime \prime}=0$. Two cases must be considered:

(i) $k-1 \geq 1$. Repeated applications of lemma 1 show again that $b_{k-1}^{\prime \prime \prime}$, $b_{k-1}^{\prime \prime}$ and $b_{k-1}^{\prime}$ can be chosen to vanish so that $b_{k-1}$ is $\gamma$-closed,

$$
b_{k-1}=\Sigma b_{J} \omega^{J}(C) .
$$


Inserting the respective forms (22) and (23) of $a_{k}$ and $b_{k-1}$ in $\gamma a_{k-1}+\delta a_{k}+$ $d b_{k-1}=0$ yields the condition that $\Sigma\left(\delta a_{J}+d b_{J}\right) \omega^{J}(C)$ should be $\gamma$-exact. But then, $\left(\delta a_{J}+d b_{J}\right)$ must vanish. Lemma 2 implies that $a_{J}$ is $\delta$-closed modulo $d, a_{J}=\delta p_{J}+d q_{J}$ (where $p_{J}$ and $q_{J}$ may be chosen to fulfill $\gamma p_{J}=0=\gamma q_{J}$ [13]). Straightforward redefinitions enable one to set $a_{J}$-and thus $a_{k^{-}}$equal to zero. One can similarily set successively $a_{k-1}, a_{k-2}, \ldots$ equal to zero, until one reaches $a_{1}$.

(ii) $k=1$. If the gauge group is semi-simple, then $a_{1}=0$. Indeed, there is no Lie algebra cohomology in degree 1 or 2 . The equation $s a+d b=0$ reduces to $\gamma a_{0}+d b_{0}=0$, which is precisely the equation studied and solved in the literature [15, 17, 18]. The coresponding solutions are (3), (7) and (9). If the gauge group has abelian factors, there are additional solutions because the Lie algebra cohomology is non trivial in degree 1 or 2 . The invariant cocycles are $k_{A}^{\Delta} C^{A}$ in degree 1 and $k_{A B}^{\Delta} C^{A} C^{B}$ in degree 2. For each of these cocycles, one may form an element $a_{1}$ by taking the product with an element of $H_{1}^{-1,4}(\delta \mid d)$, which is isomorphic to the space of non trivial conserved currents (lemma 2). The corresponding solutions are (4) and (10). This completes the proof of the theorems.

In this letter, we have settled down some old problems on the perturbative renormalization of the Yang-Mills field with a simple or semi-simple gauge group. We have shown that the inclusion of the antifields (sources for the BRST variations) does not modify the BRST cohomology in the space of integrated polynomials with ghost degree equal to 0 or 1, except for making trivial BRST invariant objects that vanish on-shell. The BRST cohomology in higher ghost degree is modified, however. We have also analyzed the case with abelian factors and have exhibited all BRST invariant integrated polynomials. These may now depend non trivially on the antifields, as in [10]. The new solutions are of mathematical interest in the sense that their understanding involve many ingredients (conserved currents, Koszul-Tate resolution ...) but we have not investigated whether they actually occur. Finally, the same techniques can be applied to the calculation of $H^{l, j}(s \mid d, \mathcal{B})$ for any ghost number $l$ or form degree $j$ and in any number of spacetime dimensions. A fuller account of our work will be reported elsewhere [13].

Useful conversations with F. Brandt, M. Dubois-Violette, M. Talon and C.M. Viallet are gratefully acknowledged. G.B. is Aspirant au Fonds National de la Recherche Scientifique (Belgium). This work has been partly supported by a FNRS research grant and by a research contract with the Commission 
of the European Communities.

\section{References}

[1] I.A. Batalin and G.A. Vilkovisky, Phys. Lett. B 102 (1982) 27 ; B 120 (1983) 166 ; Phys. Rev. D 28 (1983) 2567.

[2] J.M.L. Fisch and M. Henneaux, Commun. Math. Phys. 128 (1990) 627 ; see also J. Fisch, M. Henneaux, J. Stasheff and C. Teitelboim, Commun. Math. Phys. 127 (1989) 379 ; M. Henneaux, Nucl. Phys. B (Proc. Suppl.) 18A (1990) 47.

[3] M. Henneaux, Phys. Lett. B 313 (1993) 35.

[4] S.D. Joglekar and B.W. Lee, Ann. Phys. (NY) 97 (1976) 160.

[5] H. Kluberg-Stern and J.B. Zuber, Phys. Rev. D12 (1975) 467, 482, 3159.

[6] B.L. Voronov and I.V. Tyutin, Theor. Math. Phys. 52 (1983) 628.

[7] J. Zinn-Justin, Quantum Field Theory and Critical Phenomena, $2^{\text {nd }}$ Edition, Clarendon Press (Oxford: 1993).

[8] R. Stora, Differential Algebras in Field Theory, in Quantum Mechanics of Fundamental Systems 2, C. Teitelboim and J. Zanelli eds., Plenum Press (New York: 1989).

[9] M. Henneaux and C. Teitelboim, Quantization of Gauge Systems, Princeton University Press (Princeton: New Jersey 1992).

[10] F. Brandt, Antifield Dependence of Anomalies, preprint NIKHEF-H 93-22, (to appear in Phys. Lett. B).

[11] J. Dixon and M. Ramon Medrano, Phys. Rev. D 22 (1980) 429.

[12] G. Barnich and M. Henneaux, Phys. Lett. B 311 (1993) 123.

[13] G. Barnich and M. Henneaux, in preparation.

[14] The differential $\gamma$ was denoted by $\sigma$ in [3]. However, since the letter $\sigma$ is also used for the "quantum BRST differential" $\sigma=s+\hbar \Delta$ [9], we shall not use this notation here in order to avoid confusion.

[15] J.A. Dixon, Commun. Math. Phys. 139 (1991) 495. 
[16] The converse is not true. One may have $a \neq \gamma c+d e$ but $a=s c+d e$, for instance if $a$ vanishes with the equations of motion.

[17] F. Brandt, N. Dragon and M. Kreuzer, Nucl. Phys. B332 (1990) 224.

[18] M. Dubois-Violette, M. Henneaux, M. Talon and C.M. Viallet, Phys. Lett. B 289 (1992) 361.

[19] M. Henneaux, Commun. Math. Phys. 140 (1991) 1.

[20] G. Bandelloni, J. Math. Phys. 27 (1986) 2551 ; 28 (1987) 2775.

[21] M. Dubois-Violette, M. Talon and C.M. Viallet, Commun. Math. Phys. 102 (1985) 105. 